\title{
Prevalence of antenatal and postnatal anxiety: systematic review and meta-analysis
}

Cindy-Lee Dennis, Kobra Falah-Hassani and Rahman Shiri

\section{Background}

Maternal anxiety negatively influences child outcomes. Reliable estimates have not been established because of varying published prevalence rates.

\section{Aims \\ To establish summary estimates for the prevalence of maternal anxiety in the antenatal and postnatal periods.}

\section{Method}

We searched multiple databases including MEDLINE, Embase, and PsycINFO to identify studies published up to January 2016 with data on the prevalence of antenatal or postnatal anxiety. Data were extracted from published reports and any missing information was requested from investigators.

Estimates were pooled using random-effects meta-analyses.

\section{Results}

We reviewed 23468 abstracts, retrieved 783 articles and included 102 studies incorporating 221974 women from 34 countries. The prevalence for self-reported anxiety symptoms was $18.2 \%$ (95\% Cl 13.6-22.8) in the first trimester, 19.1\%
(95\% Cl 15.9-22.4) in the second trimester and $24.6 \%(95 \% \mathrm{Cl}$ 21.2-28.0) in the third trimester. The overall prevalence for a clinical diagnosis of any anxiety disorder was $15.2 \%$ (95\% Cl 9.0-21.4) and 4.1\% (95\% Cl 1.9-6.2) for a generalised anxiety disorder. Postnatally, the prevalence for anxiety symptoms overall at 1-24 weeks was $15.0 \%(95 \% \mathrm{Cl}$ 13.7-16.4). The prevalence for any anxiety disorder over the same period was $9.9 \%(95 \% \mathrm{Cl} 6.1-13.8)$, and $5.7 \%(95 \% \mathrm{Cl}$ 2.3-9.2) for a generalised anxiety disorder. Rates were higher in low- to middle-income countries.

\section{Conclusions}

Results suggest perinatal anxiety is highly prevalent and merits clinical attention. Research is warranted to develop evidence-based interventions.

\section{Declaration of interest}

None.

\section{Copyright and usage}

(c) The Royal College of Psychiatrists 2017.
Perinatal mental health is a leading public health issue because of its negative effect on both maternal and child outcomes and its significant economic cost to society if left untreated. ${ }^{1,2}$ A common mental health problem women experience during the perinatal (pregnancy and postpartum) period is anxiety ${ }^{3}$ and despite it being a frequent comorbidity with depression, ${ }^{4}$ it has received limited attention from researchers and health professionals. This is an important clinical omission given the ever-growing evidence indicating maternal anxiety both antenatally and postnatally may lead to serious negative outcomes. Maternal antenatal anxiety has been associated with increased childbirth fear, ${ }^{5}$ a preference for Caesarean section delivery, ${ }^{6}$ decreased effective coping strategies, ${ }^{7}$ higher rates of eating disorders ${ }^{8}$ and an increased risk for suicide. ${ }^{9}$ It also has important neonatal implications as it has been linked to increased preterm birth rates, ${ }^{10,11}$ lower Apgar scores ${ }^{12}$ and decreased birth length. ${ }^{13}$ Further, antenatal anxiety is a risk factor for poor child developmental trajectories. ${ }^{14}$ In a study conducted in the Netherlands, antenatal anxiety early in pregnancy significantly increased the risk for cognitive disorders in children at 14 and 15 years of age. ${ }^{15}$ In the same population, hierarchical multiple regression analyses showed that maternal anxiety at 12-22 weeks' gestation explained 22\%, 15\% and 9\% of the variance in cross-situational attention-deficit hyperactivity disorder symptoms, externalising problems and self-reported anxiety, respectively, among Dutch children aged 8 and 9 years. ${ }^{16}$ The link between antenatal anxiety and behavioural/emotional problems in children at 4 years of age after adjusting for covariates has also been reported in a UK study. ${ }^{17}$ More recently, maternal antenatal anxiety was associated with an increased risk of child attention problems after accounting for confounders. ${ }^{18}$ Similar adverse effects have been found for maternal postnatal anxiety, which has been associated with negative and disengaged parenting ${ }^{19-21}$ and overcontrolling maternal behaviours that increase the likelihood of internalising and externalising difficulties in the child. ${ }^{20,22,23}$ The emergent evidence highlights the need for early identification of maternal anxiety across the perinatal period and the provision of effective treatment. However, reliable estimates of maternal anxiety to guide clinical interventions are unknown because of widely varying published prevalence rates. The aim of this systematic review was to establish summary estimates for the prevalence of maternal anxiety in the antenatal and postnatal periods.

\section{Method}

\section{Search strategy and study eligibility}

The protocol and reporting of the results of this systematic review and meta-analysis were based on PRISMA guidelines. ${ }^{24}$ Comprehensive literature searches were conducted in MEDLINE, Embase, PsycINFO, Cumulative Index to Nursing and Allied Health Literature, Web of Science, Scopus, ResearchGate and Google Scholar from 1950 until 13 January 2016 using predefined key terms (online Table DS1) such as (postpartum OR puerperium OR pregnancy OR gestation OR postbirth OR post-birth OR antenatal OR prenatal OR postnatal) AND (mood disorders OR depressive disorder OR depression OR depressive symptoms $\mathrm{OR}$ anxiety disorders $\mathrm{OR}$ anxiety). We used $\mathrm{MeSH}$ terms and key words in MEDLINE and Emtree terms and key words in Embase. The titles and abstracts of all identified citations were screened for relevance and the full text of potentially relevant articles were obtained and assessed for eligibility. In addition, the reference lists of relevant articles were hand searched.

Studies were eligible for inclusion if they: (a) included women who were 16 years or older; (b) assessed for antenatal or postnatal anxiety using a validated diagnostic or self-report instrument; 
(c) reported the results of peer-reviewed research based on crosssectional or cohort studies; and (d) provided data in order to estimate the prevalence of anxiety. Studies were excluded if they: (a) were conducted among self-selected volunteers; (b) recruited high-risk women; (c) reported results for only a subsample of a study population; (d) reported duplicate data from a single database; (e) reported only mean data; (e) reported combined prevalence for depression and anxiety; or (f) did not report a cut-off point for anxiety. We contacted over 70 authors for additional information, particularly those who reported only mean data, no cut-off data or had missing information, with approximately a third providing us with additional results. For studies with duplicate data from a single database, we selected the study with the larger sample size.

\section{Data extraction and quality assessment}

We extracted individual details of the included studies such as year of publication, study population, recruitment method, sample size used in the analysis, measure of anxiety, cut-off points, timing of assessments and prevalence of anxiety variously defined. The risk of bias in the included studies was independently rated by two reviewers (K.F.-H. and R.S.) using criteria adapted from the Effective Public Health Practice Project Quality Assessment Tool for observational studies. ${ }^{25}$ Three domains were assessed: selection bias, detection bias and attrition bias. Selection bias was classified as: (a) low: likely to be representative of the target population or subgroup of the target population (i.e. specific age group or geographic area) and response rate was $80 \%$ or higher; (b) moderate: likely to be somewhat representative of the target population or a restricted subgroup of the target population and response rate was $60-79 \%$; or (c) high: target population was self-referred/volunteers, or response rate was less than $60 \%$. Detection bias was classified as follows: (a) low: the outcome was defined by clinical diagnosis; (b) moderate: the outcome was assessed by a validated questionnaire; or (c) high: the outcome was self-reported. Finally, attrition bias was classified as follows: (a) low: follow-up participation rate was more than $80 \%$ or missing data was less than 20\%; (b) moderate: follow-up participation rate was $60-79 \%$ or missing data was $20-40 \%$; or (c) high: follow-up participation rate was less than $60 \%$ or missing data was more than $40 \%$. Any disagreements in quality ratings were resolved by discussion (K.F.-H., R.S.), and if necessary with the involvement of another author (C.-L.D.).

\section{Data synthesis and analysis}

Many studies reported an estimate for the prevalence of antenatal or postnatal anxiety for more than one time point for the same participants. In order to include each study with multiple timepoints only once in a specific meta-analysis, an overall prevalence of antenatal or postnatal anxiety was estimated using an average sample size and an average number of events (for example estimate for the 1-24 weeks' postnatal anxiety symptoms). The prospective cohort studies included in the current meta-analysis determined the prevalence of anxiety rather than the incidence of anxiety. We therefore combined both cross-sectional and cohort studies in a single analysis. Anxiety was assessed using diverse measures, cut-off scores and perinatal time periods. We performed meta-analyses based on the following anxiety categories: (a) selfreported state anxiety symptoms, (b) self-reported trait anxiety, (c) clinical diagnosis of any anxiety disorder, and (d) clinical diagnosis of generalised anxiety disorder. We further performed analyses according to pregnancy trimester and postpartum time period. We used a random-effects meta-analysis to combine the estimates of different studies. ${ }^{26}$ The presence of heterogeneity across the studies was assessed using the $I^{2}$-statistic. ${ }^{27}$ An $I^{2}$-statistic less than $25 \%$ indicates small inconsistency and more than $50 \%$ indicates large inconsistency. ${ }^{27}$ We used meta-regression to assess the differences between subgroups. ${ }^{26}$ We performed subgroup analyses according to year of publication $(\geqslant 2009 \mathrm{v}$. $\leqslant 2010$ ), income of study country based on World Bank categories (low to middle income $v$. high income), selection bias and attrition bias. Stata (version 13) was used for the meta-analyses.

\section{Results}

\section{Study characteristics}

The study selection process is presented in Fig. 1. The literature search yielded 23468 unique references, of which 22685 were excluded following title and abstract screening. Overall, 783 full papers were retrieved and assessed. Of these, 183 papers were relevant following full-text screening: 174 were identified from searches of electronic databases and 9 from hand searches of references. From these 183 studies, a further 81 were excluded primarily for only having mean anxiety scores $(n=35)$ and volunteer samples $(n=18)$. In total, 102 studies on antenatal or postnatal anxiety were included in the meta-analyses with assistance from 26 authors who were contacted and provided additional information to allow their studies to be incorporated (see Acknowledgements).

Characteristics of the included studies are provided in online Table DS2. In total, 70 studies provided data on the prevalence of antenatal anxiety and 57 studies provided data related to postnatal anxiety. The studies were conducted in 34 different countries spanning six continents and included 221974 women.

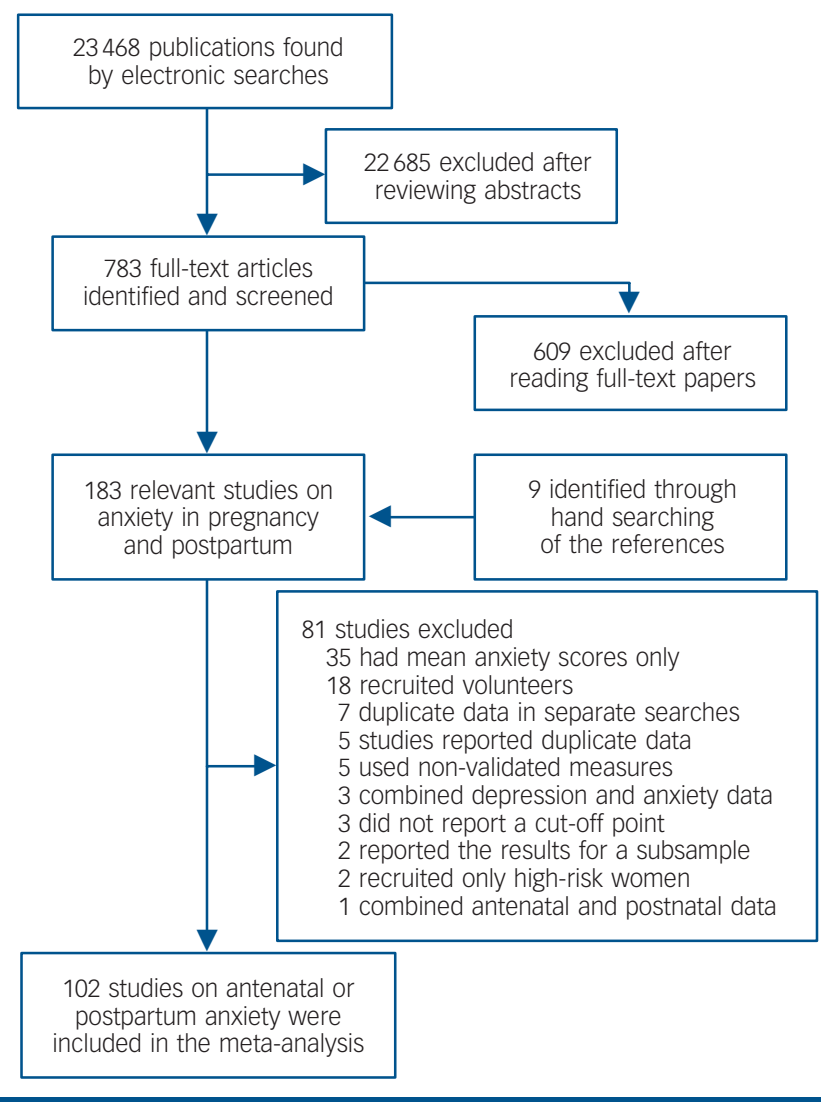

Fig. 1 Flow diagram for identifying studies on the prevalence of antenatal and postnatal anxiety. 
The countries with the largest number of included studies comprised the USA $(n=19)$, Australia $(n=11)$, Brazil $(n=9)$, Canada $(n=8)$, France $(n=4)$, Netherlands $(n=4)$, Norway $(n=4)$, UK $(n=4)$, Germany $(n=3)$ and Sweden $(n=3)$. Ten countries from the Asian continent provided data (Bangladesh, China, Hong Kong, Israel, Japan, Jordan, Malaysia, Saudi Arabia, Singapore and Vietnam) as did four countries from Africa (Ghana, Nigeria, South Africa and Tanzania). In total, there were 24 countries classified as low to middle income using World Bank categories. The majority of studies used the self-report State-Trait Anxiety Inventory (STAI) to measure state anxiety symptoms $(n=51)$ or trait anxiety $(n=24)$. The most common diagnostic interviews to assess for any anxiety disorders or generalised anxiety disorder were the Mini-International Neuropsychiatric Interview $(n=6)$, Composite International Diagnostic Interview $(n=5)$ and Structural Clinical Interview for DSM $(n=5)$. When evaluated by the modified Effective Public Health Practice Project Quality Assessment Tool, eight studies were rated as having low risk of selection bias, 69 as having moderate risk and 25 studies as having high risk (Table 2). In total, 17 studies were rated as having low risk of detection bias, 85 as having moderate risk, and none as having high risk. For attrition bias, 77 studies were rated as low risk, 17 as moderate risk and 8 as high risk.

\section{Prevalence of antenatal anxiety}

Meta-analytic pooling of the estimates yielded the prevalence of self-reported anxiety symptoms to be $18.2 \%$ (95\% CI $13.6-22.8$, 10 studies, $n=10577)^{6,12,28-35}$ for the first trimester, $19.1 \%$ (95\% CI 15.9-22.4, 17 studies, $n=24499)^{10,12,29,31,32,34-45}$ for the second trimester and $24.6 \%$ (95\% CI 21.2-28.0, 33 studies, $n=116720)^{3,5,7,12,13,29-32,34,35,37,40,43,44,46-63}$ for the third trimester (Table 1 and Fig. 2). The overall pooled prevalence for self-reported anxiety symptoms across the three trimesters was $22.9 \%$ (95\% CI 20.5-25.2, 52 studies, $n=142833)$. The prevalence for selfreported trait anxiety was $29.1 \%$ (95\% CI 11.7-46.4, 4 studies, $n=2388$ ) for the first trimester, and 32.5\% (95\% CI 27.6-37.4, 12 studies, $n=5568)$ for the third trimester. The prevalence for a clinical diagnosis of any anxiety disorder was $18.0 \%(95 \%$ CI $15.0-21.1,2$ studies, $n=615$ ) for the first trimester, $15.2 \%$ (95\% CI 3.6-26.7, 4 studies, $n=3002$ ) for the second trimester and $15.4 \%$ (95\% CI 5.1-25.6, 4 studies, $n=1603$ ) for the third trimester. The prevalence of a clinical diagnosis of a generalised anxiety disorder was $5.3 \%$ (95\% CI $1.5-9.1,3$ studies, $n=3338$ ) for the first trimester, $0.3 \%$ (95 CI \% $0.1-0.6,2$ studies, $n=1862)$ and $4.1 \%$ (95\% CI 1.0-7.2, 4 studies, $n=1455)$ for the second and third trimester, respectively. Overall, the prevalence of any anxiety disorder across the three trimesters was $15.2 \%$ (95\% CI 9.0-21.4, 9 studies, $n=4648$, Table 1 and online Fig. DS1) and that of a generalised anxiety disorder was 4.1\% (95\% CI 1.9-6.2, 10 studies, $n=6910$, Table 1 and online Fig. DS2).

\section{Prevalence of postnatal anxiety}

The prevalence of self-reported anxiety symptoms was $17.8 \%(95 \%$ CI $14.2-21.4,14$ studies, $n=10928$ ) at $1-4$ weeks postpartum, $14.9 \%$ (95\% CI 12.3-17.5, 22 studies, $n=19158$ ) at 5-12 weeks postpartum, $15.0 \%$ (95\% CI 13.7-16.4, 39 studies, $n=145293$ ) at 1-24 weeks postpartum, and $14.8 \%$ (95\% CI 10.9-18.8, 7 studies, $n=11528$ ) at $>24$ weeks postpartum (Table 2 and online Fig. DS3). The prevalence of having a clinical diagnosis of any anxiety disorder was $9.6 \%(95 \%$ CI $3.4-15.9,5$ studies, $n=2712$ ) at $5-12$ weeks postpartum, 9.9\% (95\% CI 6.1-13.8, 9 studies, $n=28495)$ at $1-24$ weeks postpartum and $9.3 \%(95 \%$ CI 5.5-13.1, 5 studies, $n=28244$ ) at $>24$ weeks postpartum (Table

\begin{tabular}{|c|c|c|c|c|c|c|c|c|}
\hline \multirow[b]{2}{*}{$\begin{array}{l}\text { Time period, measure } \\
\text { and outcome }\end{array}$} & \multicolumn{4}{|c|}{ All studies } & \multicolumn{4}{|c|}{ Studies without high risk of selection/attrition bias } \\
\hline & $\begin{array}{c}\text { Studies, } \\
n\end{array}$ & Sample & $\begin{array}{l}\text { Prevalence, } \\
\%(95 \% \mathrm{Cl})\end{array}$ & $I^{2}, \%$ & $\begin{array}{c}\text { Studies, } \\
n\end{array}$ & Sample & $\begin{array}{l}\text { Prevalence, } \\
\%(95 \% \mathrm{Cl})\end{array}$ & $I^{2}, \%$ \\
\hline \multirow{2}{*}{\multicolumn{9}{|c|}{$\begin{array}{l}\text { First trimester } \\
\text { Self-report }\end{array}$}} \\
\hline & & & & & & & & \\
\hline Trait anxiety & 4 & 2388 & $29.1(11.7-46.4)$ & 99.0 & 2 & 1532 & $38.4(36.1-40.7)$ & 99.6 \\
\hline Anxiety symptoms & 10 & 10577 & $18.2(13.6-22.8)$ & 97.3 & 9 & 8974 & $19.1(13.3-24.8)$ & 97.6 \\
\hline \multicolumn{9}{|l|}{ Clinical diagnosis } \\
\hline Any anxiety disorder & 2 & 615 & $18.0(15.0-21.1)$ & 99.7 & 2 & 615 & $18.0(15.0-21.1)$ & 99.7 \\
\hline Generalised anxiety disorder & 3 & 3338 & $5.3(1.5-9.1)$ & 94.7 & 3 & 3338 & $5.3(1.5-9.1)$ & 94.7 \\
\hline \multirow{2}{*}{\multicolumn{9}{|c|}{$\begin{array}{l}\text { Second trimester } \\
\text { Self-report }\end{array}$}} \\
\hline & & & & & & & & \\
\hline Trait anxiety & 1 & - & - & - & 1 & - & - & - \\
\hline Anxiety symptoms & 17 & 24499 & $19.1(15.9-22.4)$ & 97.9 & 13 & 18430 & $19.4(15.7-23.2)$ & 97.3 \\
\hline \multicolumn{9}{|l|}{ Clinical diagnosis } \\
\hline Any anxiety disorder & 4 & 3002 & $15.2(3.6-26.7)$ & 98.7 & 4 & 3002 & $15.2(3.6-26.7)$ & 98.7 \\
\hline Generalised anxiety disorder & 2 & 1862 & $0.3(0.1-0.6)$ & 97.3 & 2 & 1862 & $0.3(0.1-0.6)$ & 97.3 \\
\hline \multicolumn{9}{|l|}{ Third trimester } \\
\hline \multicolumn{9}{|l|}{ Self-report } \\
\hline Trait anxiety & 12 & 5568 & $32.5(27.6-37.4)$ & 92.5 & 8 & 4168 & $31.4(25.9-36.9)$ & 92.4 \\
\hline Anxiety symptoms & 33 & 116720 & $24.6(21.2-28.0)$ & 98.9 & 22 & 16120 & $23.4(19.9-26.9)$ & 95.9 \\
\hline \multicolumn{9}{|l|}{ Clinical diagnosis } \\
\hline Any anxiety disorder & 4 & 1603 & $15.4(5.1-25.6)$ & 97.6 & 2 & 615 & $14.2(11.5-16.9)$ & 99.6 \\
\hline Generalised anxiety disorder & 4 & 1455 & $4.1(1.0-7.2)$ & 92.5 & 3 & 958 & $2.3(0.2-4.4)$ & 80.1 \\
\hline \multirow{2}{*}{\multicolumn{9}{|c|}{$\begin{array}{l}\text { First, second or third trimester } \\
\text { Self-report }\end{array}$}} \\
\hline & & & & & & & & \\
\hline Trait anxiety & 18 & 8086 & $31.5(26.3-36.7)$ & 96.3 & 11 & 5372 & $34.3(28.5-40.1)$ & 94.9 \\
\hline Anxiety symptoms & 52 & 142833 & $22.9(20.5-25.2)$ & 99.0 & 35 & 35656 & $22.4(19.6-25.1)$ & 97.8 \\
\hline \multicolumn{9}{|l|}{ Clinical diagnosis } \\
\hline Any anxiety disorder & 9 & 4648 & $15.2(9.0-21.4)$ & 97.7 & 6 & 3560 & $14.8(6.4-23.3)$ & 98.0 \\
\hline Generalised anxiety disorder & 10 & 6910 & $4.1(1.9-6.2)$ & 97.3 & 9 & 6413 & $3.6(1.4-5.7)$ & 97.3 \\
\hline
\end{tabular}


First trimester Betts et al (2014) ${ }^{28}$ Huizink et al (2014) ${ }^{29}$ Meijer et al (2014) ${ }^{30}$ Rubertsson et al (2014) Makara-Studzinska et al (2013) Figueiredo \& Conde (2011) ${ }^{32}$ Goebert et al (2007) ${ }^{33}$ Lee et al (2007) Berle et al (2005) ${ }^{12}$ Rondo et al $(2003)^{35}$ Subtotal $\left(I^{2}=97 \%, P=0.000\right)$

Second trimester Rosenthal et al (2015) ${ }^{36}$ Vilela et al (2015) ${ }^{37}$ Huizink et al (2014) ${ }^{29}$ Khashan et al (2014) ${ }^{38}$ Fadzil et al (2013) ${ }^{39}$ Makara-Studzinska et al (2013) Roos et al (2013) ${ }^{40}$ Figueiredo \& Conde $(2011)^{32}$ Ibanez et al (2012) ${ }^{10}$ Couto et al (2009) ${ }^{41}$ van Batenburg-Eddes et al (2009) ${ }^{42}$ Lee et al (2007) ${ }^{34}$ Shi et al $(2007)^{43}$ Berle et al (2005) ${ }^{12}$ Heron et al (2004) $)^{44}$ Dole et al $(2003)^{45}$ Rondo et al (2003) $)^{35}$ Subtotal $\left(I^{2}=98 \%, P=0.000\right)$

Third trimester Aaron et al (2015) $)^{46}$ Bjork et al (2015) ${ }^{47}$ Cheng et al (2015) ${ }^{48}$ Dubber et al (2015) Ibanex et al (2015) Pazzagl et al (2015) Pisoni et al (2016) ${ }^{52}$ Vilela et al $(2015)^{37}$ Broekman et al (2014) ${ }^{13}$ Ferreira et al (2014) $)^{53}$ Garthus-Niegel et al (2014) ${ }^{3}$ Huizink et al (2014) ${ }^{29}$ Meijer et al (2014) Jokic-Bergic et al (2014) ${ }^{54}$ Tan et al (2014) 55 Verreault et al (2014) ${ }^{56}$ Bindt et al (2013) George et al (2013) ${ }^{7}$ Makara-Studzinska et al (2013) Roos et al $(2013)^{40}$ Figueiredo \& Conde $(2011)^{32}$ Mohammad et al (2011) Hall et al (2009) Dipietro et al (2008) ${ }^{59}$ Keeton et al $(2008)^{60}$ Lee et al (2007) ${ }^{34}$ Shi et al $(2007)^{43}$ Bene et al (2005) 12 Heron et al (2004) ${ }^{44}$ Rondo et al (2003) $)^{35}$ Ross et al (2003) ${ }^{61}$ Teixeira et al (1999) ${ }^{62}$ Crandon et al (1979) ${ }^{63}$ Subtotal $\left(I^{2}=99 \%, P=0.000\right)$

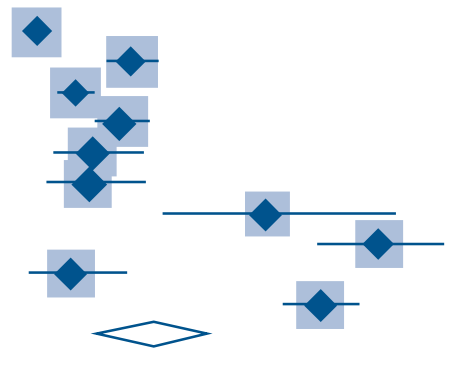

$0.089(0.081-0.097)$

$0.165(0.144-0.189)$

$0.120(0.105-0.137)$

$0.156(0.134-0.181)$

$0.134(0.101-0.176)$

$0.131(0.095-0.177)$

$0.274(0.190-0.377)$

$0.364(0.314-0.417)$

$0.116(0.082-0.162)$

$0.317(0.287-0.349)$

$0.182(0.136-0.228)$

$0.200(0.179-0.223)$

$0.439(0.371-0.509)$

$0.133(0.114-0.155)$

$0.201(0.190-0.212)$

$0.234(0.178-0.302)$

$0.073(0.049-0.108)$

$0.543(0.448-0.635)$

$0.123(0.089-0.169)$

$0.079(0.067-0.092)$

$0.333(0.255-0.422)$

$0.087(0.076-0.099)$

$0.322(0.275-0.374)$

$0.050(0.034-0.072)$

0.075 (0.047-0.117)

$0.146(0.139-0.154)$

$0.179(0.161-0.197)$

$0.265(0.236-0.295)$

$0.191(0.159-0.224)$

$0.274(0.201-0.363)$

$0.058(0.056-0.059)$

$0.263(0.237-0.290)$

$0.291(0.188-0.421)$

$0.211(0.192-0.230)$

$0.304(0.237-0.379)$

$0.270(0.154-0.430)$

$0.485(0.416-0.554)$

$0.303(0.275-0.331)$

$0.585(0.511-0.655)$

$0.084(0.072-0.098)$

$0.154(0.133-0.188)$

$0.140(0.124-0.158)$

$0.350(0.287-0.418)$

$0.611(0.518-0.695)$

$0.266(0.224-0.314)$

$0.142(0.119-0.170)$

$0.185(0.150-0.226)$

$0.146(0.112-0.190)$

$0.486(0.392-0.580)$

$0.181(0.139-0.232)$

$0.144(0.112-0.185)$

$0.294(0.260-0.330)$

$0.285(0.216-0.365)$

$0.359(0.288-0.438)$

$0.358(0.209-0.411)$

$0.066(0.047-0.091$ (

$0.123(0.084-0.175)$

$0.156(0.148-0.164)$

$0.292(0.263-0.324)$

$0.100(0.062-0.158)$

$0.150(0.093-0.233)$

$0.233(0.172-0.308)$

$0.246(0.212-0.280)$
10.81

10.57

10.70

10.54

10.11

9.98

7.38

9.54

10.03

10.33

100.00

6.30

5.00

6.33

6.45

5.22

6.18

4.16

5.91

6.43

4.51

6.45

5.62

6.36

6.05

6.48

6.37

6.17

100.00

2.78

3.32

3.25

2.34

3.28

2.89

2.09

2.91

3.24

2.88

3.30

3.27

3.29

2.95

2.69

3.13

3.25

3.18

3.18

2.63

3.12

3.19

3.20

2.85

2.84

3.08

3.27

3.13

3.31

3.23

3.11

2.91

2.92

100.00

\begin{tabular}{|c|c|c|c|c|c|c|c|}
\hline & $T$ & $T$ & $T$ & $T$ & $T$ & 1 & 1 \\
\hline 0 & 0.1 & 0.2 & 0.3 & 0.4 & 0.5 & 0.6 & 0.7 \\
\hline
\end{tabular}

Fig. 2 Prevalence of antenatal anxiety symptoms. 
2 and online Fig. DS1).The prevalence of a generalised anxiety disorder was $6.7 \%(95 \%$ CI $0.6-12.7,4$ studies, $n=1979)$ at $5-12$ weeks postpartum, 5.7\% (95\% CI 2.3-9.2, 6 studies, $n=2667)$ at 1-24 weeks postpartum, and 4.2\% (95\% CI 1.5-6.9, 4 studies, $n=1950)$ at $>24$ weeks postpartum (Table 2 and online Fig. DS2).

\section{Sensitivity and subgroup analyses}

Excluding studies with high risk of selection or attrition bias did not change markedly the estimates for the prevalence of antenatal and postnatal anxiety symptoms, any anxiety disorder or a generalised anxiety disorder (Tables 1 and 2). The prevalence of antenatal and postnatal anxiety symptoms as well as that of antenatal and postnatal anxiety disorder did not differ with regard to year of publication (>2010 v. <2009), selection bias and attrition bias (Table 3). However, the prevalence of antenatal anxiety symptoms across all trimesters was significantly higher in low- to middle-income countries (34.4\%, 95\% CI 25.0-43.8, 13 studies, $n=5089$ ) in comparison with high-income countries (19.4\% 95\% CI 17.0-21.8, 39 studies, $n=137744$ ). The prevalence of postnatal anxiety symptoms across the first 6 months postpartum was also significantly higher in low- to middle-income countries $(25.9 \%$, 95\% CI $13.7-$ 38.1, 5 studies, $n=2159$ ) in comparison with high-income countries (13.7\%, 95\% CI 12.3-15.0, 34 studies, $n=143$ 134) (Table 3). Studies with moderate or high risk of selection bias may have overestimated the prevalence of antenatal and postnatal anxiety symptoms.

\section{Discussion}

\section{Main findings}

This is the first systematic review and meta-analysis to estimate the prevalence of antenatal and postnatal anxiety. Included were
102 studies involving 221974 women from 34 countries with 26 study authors providing additional information to promote the comprehensiveness and generalisability of the meta-analytic results. Overall, the prevalence rate for self-report anxiety symptoms in the first trimester was $18.2 \%$ increasing as the pregnancy progressed to $24.6 \%$ in the third trimester. The prevalence of anxiety symptoms across the three trimesters was $22.9 \%$. Postnatally, $17.8 \%$ of women experienced significant anxiety symptoms in the first 4 weeks following childbirth but rates stabilised to approximately $15 \%$ thereafter. When diagnostic interviews were employed, the prevalence rate for any anxiety disorder during the first trimester was $18 \%$ decreasing marginally to approximately $15 \%$ in the final two trimesters of pregnancy. The prevalence of any anxiety disorder continued to decrease postnatally and ranged from 9.3 to $9.9 \%$ across the first year. As expected, rates for a generalised anxiety disorder were lower at $4 \%$ across the pregnancy and increased slightly to $4.2-5.7 \%$ postnatally. Overall, our findings demonstrate anxiety is a common mental health problem among pregnant and postpartum women internationally and that rates are significantly higher in this maternal population than in the general adult population. ${ }^{64,65}$

In interpreting the results, it is important to note that the majority of studies assessed anxiety using self-report instruments that measured anxiety symptoms rather than gold-standard diagnostic clinical interviews for various anxiety disorders. Although the sensitivity and specificity of these self-report instruments vary substantially, the most frequently used measure in this review was the STAI, a finding consistent with previous research. ${ }^{66}$ Self-report measures do have limitations, such as potentially inflated prevalence estimates, but they also have high clinical utility in obstetric/midwifery, public health and primary care practices, where the majority of perinatal mental health

\begin{tabular}{|c|c|c|c|c|c|c|c|c|}
\hline \multirow[b]{2}{*}{$\begin{array}{l}\text { Time period, measure } \\
\text { and outcome }\end{array}$} & \multicolumn{4}{|c|}{ All studies } & \multicolumn{4}{|c|}{ Studies without high risk of selection/attrition bias } \\
\hline & $\begin{array}{c}\text { Studies, } \\
n\end{array}$ & Sample & $\begin{array}{l}\text { Prevalence, } \\
\%(95 \% \mathrm{Cl})\end{array}$ & $1^{2}, \%$ & $\begin{array}{c}\text { Studies, } \\
n\end{array}$ & Sample & $\begin{array}{l}\text { Prevalence, } \\
\%(95 \% \mathrm{Cl})\end{array}$ & $I^{2}, \%$ \\
\hline \multirow{2}{*}{\multicolumn{9}{|c|}{$\begin{array}{l}\text { 1-4 weeks postpartum } \\
\text { Self-report }\end{array}$}} \\
\hline & & & & & & & & \\
\hline Trait anxiety & 6 & 2724 & $23.1(14.5-31.7)$ & 97.1 & 6 & 2724 & $23.1(14.5-31.7)$ & 97.1 \\
\hline Anxiety symptoms & 14 & 10928 & $17.8(14.2-21.4)$ & 96.1 & 12 & 10065 & $17.8(13.9-21.6)$ & 96.2 \\
\hline \multicolumn{9}{|l|}{ Clinical diagnosis } \\
\hline Any anxiety disorder & 0 & - & - & - & - & - & - & - \\
\hline Generalised anxiety disorder & 0 & - & - & - & - & - & - & - \\
\hline \multicolumn{9}{|l|}{ 5-12 weeks postpartum } \\
\hline \multicolumn{9}{|l|}{ Self-report } \\
\hline Trait anxiety & 5 & 1260 & $23.4(13.8-33.0)$ & 92.8 & 4 & 1140 & $23.1(11.4-34.8)$ & 94.6 \\
\hline Anxiety symptoms & 22 & 19158 & $14.9(12.3-17.5)$ & 97.1 & 16 & 14024 & $15.2(11.5-18.9)$ & 97.5 \\
\hline \multicolumn{9}{|l|}{ Clinical diagnosis } \\
\hline Any anxiety disorder & 5 & 2712 & $9.6(3.4-15.9)$ & 97.6 & 4 & 2413 & $11.3(2.6-19.9)$ & 98.1 \\
\hline Generalised anxiety disorder & 4 & 1979 & $6.7(0.6-12.7)$ & 97.8 & 4 & 1979 & $6.7(0.6-12.7)$ & 97.8 \\
\hline \multicolumn{9}{|l|}{ 1-24 weeks postpartum } \\
\hline \multicolumn{9}{|l|}{ Self-report } \\
\hline Trait anxiety & 10 & 3533 & $23.2(16.0-30.4)$ & 96.6 & 8 & 3313 & $22.8(14.6-31.0)$ & 97.3 \\
\hline Anxiety symptoms & 39 & 145293 & $15.0(13.7-16.4)$ & 98.5 & 26 & 45104 & $17.2(14.3-20.0)$ & 98.8 \\
\hline \multicolumn{9}{|l|}{ Clinical diagnosis } \\
\hline Any anxiety disorder & 9 & 28495 & $9.9(6.1-13.8)$ & 97.8 & 7 & 28096 & $9.9(5.4-14.4)$ & 98.2 \\
\hline Generalised anxiety disorder & 6 & 2667 & $5.7(2.3-9.2)$ & 94.5 & 6 & 2667 & $5.7(2.3-9.2)$ & 94.5 \\
\hline \multirow{2}{*}{\multicolumn{9}{|c|}{$\begin{array}{l}>24 \text { weeks postpartum } \\
\text { Self-report }\end{array}$}} \\
\hline & & \\
\hline Trait anxiety & 1 & & & & & & & \\
\hline Anxiety symptoms & 7 & 11528 & $14.8(10.9-18.8)$ & 95.9 & 5 & 9714 & $11.5(8.2-14.8)$ & 89.2 \\
\hline \multicolumn{9}{|l|}{ Clinical diagnosis } \\
\hline Any anxiety disorder & 5 & 28244 & $9.3(5.5-13.1)$ & 98.0 & 5 & 28244 & $9.3(5.5-13.1)$ & 98.0 \\
\hline Generalised anxiety disorder & 4 & 1950 & $4.2(1.5-6.9)$ & 89.3 & 4 & 1950 & $4.2(1.5-6.9)$ & 89.3 \\
\hline
\end{tabular}




\begin{tabular}{|c|c|c|c|c|c|c|c|c|}
\hline & \multicolumn{4}{|c|}{ Anxiety symptoms } & \multicolumn{4}{|c|}{ Any anxiety disorder } \\
\hline & $\begin{array}{c}\text { Studies, } \\
n\end{array}$ & Sample & $\begin{array}{l}\text { Prevalence, } \\
\%(95 \% \mathrm{Cl})\end{array}$ & $P$ & $\begin{array}{c}\text { Studies, } \\
n\end{array}$ & Sample & $\begin{array}{l}\text { Prevalence, } \\
\%(95 \% \mathrm{Cl})\end{array}$ & $P$ \\
\hline \multicolumn{9}{|c|}{$\begin{array}{l}\text { Antenatal (first, second } \\
\text { or third trimesters) }\end{array}$} \\
\hline Publication year & & & & 0.99 & & & & 0.15 \\
\hline$\leqslant 2009$ & 20 & 19193 & $23.2(18.9-27.5)$ & & 5 & 3437 & $19.5(8.3-30.8)$ & \\
\hline$\geqslant 2010$ & 32 & 123640 & $22.6(19.8-25.4)$ & & 4 & 1211 & $10.0(3.7-16.2)$ & \\
\hline Country income & & & & 0.001 & & & & 0.53 \\
\hline Low to middle & 13 & 5089 & $34.4(25.0-43.8)$ & & 3 & 1245 & $18.2(1.7-34.8)$ & \\
\hline High & 39 & 137744 & $19.4(17.0-21.8)$ & & 6 & 3403 & $13.4(8.2-18.7)$ & \\
\hline Selection bias & & & & 0.11 & & & & 0.55 \\
\hline Low & 4 & 13034 & $15.3(11.2-19.3)$ & & 2 & 1284 & $22.8(20.6-25.1)$ & \\
\hline Moderate & 34 & 28376 & $22.1(19.0-25.1)$ & & 4 & 2276 & $10.5(5.5-15.6)$ & \\
\hline High & 14 & 101423 & $27.5(21.9-33.1)$ & & 3 & 1088 & $16.2(1.1-31.4)$ & \\
\hline Attrition bias & & & & 0.23 & & & & - \\
\hline Low & 41 & 122748 & $24.4(21.1-27.7)$ & & 8 & 4548 & $14.6(8.1-21.2)$ & \\
\hline Moderate or high & 11 & 20085 & $16.3(13.3-19.2)$ & & 1 & - & - & \\
\hline \multicolumn{9}{|l|}{ Postnatal (0-24 weeks) } \\
\hline Publication year & & & & 0.92 & & & & 0.78 \\
\hline$\leqslant 2009$ & 16 & 15832 & $15.6(12.8-18.3)$ & & 4 & 26657 & $8.1(3.9-12.3)$ & \\
\hline$\geqslant 2010$ & 23 & 129461 & $14.9(13.3-16.6)$ & & 5 & 1838 & $10.8(4.3-17.3)$ & \\
\hline Country income & & & & 0.04 & & & & - \\
\hline Low to middle & 5 & 2159 & $25.9(13.7-38.1)$ & & 1 & 871 & - & \\
\hline High & 34 & 143134 & $13.7(12.3-15.0)$ & & 8 & 27624 & $8.4(5.3-11.5)$ & \\
\hline Selection bias & & & & 0.60 & & & & 0.57 \\
\hline Low & 3 & 12930 & $9.2(4.7-13.8)$ & & 1 & 871 & - & \\
\hline Moderate & 25 & 36325 & $17.3(14.2-20.5)$ & & 6 & 27225 & $8.2(4.6-11.8)$ & \\
\hline High & 11 & 96038 & $15.1(12.0-18.2)$ & & 2 & 399 & $4.4(2.4-6.4)$ & \\
\hline Attrition bias & & & & 0.41 & & & & 0.91 \\
\hline Low & 20 & 101650 & $16.4(13.5-19.3)$ & & 7 & 28096 & $9.9(5.4-14.4)$ & \\
\hline Moderate & 15 & 37971 & $17.3(13.9-20.6)$ & & 2 & 399 & $4.4(2.4-6.4)$ & \\
\hline High & 4 & 5672 & $8.7(5.8-11.6)$ & & 0 & - & - & \\
\hline
\end{tabular}

problems are managed. Health professionals in these settings often have limited clinical expertise and time for diagnostic interviews and with research clearly suggesting informal surveillance misses at least $50 \%$ of cases, ${ }^{67}$ self-report measures are crucial for systematic case identification. To reflect the heterogeneity of the measures included in this meta-analysis, a range of prevalence estimates was reported in addition to a single estimate.

\section{Prevalence rates in different countries}

The varying prevalence rates between the included studies may further be attributed to diverse settings, recruitment strategies, inclusion and exclusion criteria, data-collection methods and follow-up time periods. Language or translation complexities and variations in conveying psychiatric symptoms are other potential methodological issues. ${ }^{68}$ However, there might also be real differences in prevalence rates because of cultural influences. This may partially explain the significantly higher self-reported anxiety rates found both antenatally and postnatally between low- to middle-income countries and high-income countries in this review. Whereas genetic and neurobiological determinants are probably evenly distributed among all women and are relevant aetiological factors, ${ }^{68}$ the distribution of anxiety may be different across cultures, supporting environmental influences in the aetiology of perinatal anxiety. Our results are consistent with another systematic review that found rates of 'common perinatal mental disorders' among World Bank categorised low- and middle-income countries were significantly greater than those reported in high-income countries. ${ }^{69}$ Together, these findings challenge the idea that women's mental health is protected by culturally prescribed traditional postpartum rituals. There is also growing evidence that many risk factors for perinatal mental health in low- and middle-income countries may be influenced by conditions that transcend the woman's control. These risk factors include gender-based issues such as bias against female infants, restricted housework and infant care roles, and excessive unpaid workloads especially in multigenerational households. ${ }^{69}$ Perinatal mental health in low- and middle-income countries has only recently started to receive attention partially because of previous priorities targeting maternal mortality. As such, in this review there were considerably more studies conducted in high-income countries than in low- to middle-income countries. High-quality research addressing perinatal mental health in low- and middle-income countries is warranted to guide clinical interventions and policies.

\section{Prevalence rates over time}

Although the media often portrays an increase in anxiety prevalence rates, there is no reliable evidence to support the notion that mental disorders in general are rising. ${ }^{70,71}$ This is consistent with our results where we found no difference in prevalence rates for anxiety symptoms or disorders between studies published before 2010 and those published afterwards. However, rates of mental health treatment seeking have increased and may be the reason for the general perception that anxiety is more prevalent. ${ }^{68}$ Despite improvements in treatment, anxiety remains undetected and untreated in the general population ${ }^{72}$ and in perinatal women. To date, perinatal mental health research and clinical practice has disproportionately targeted depression 
with limited attention on anxiety. This is an important omission given a recent review indicating clinically relevant associations between antenatal anxiety and adverse child outcomes, with a 10 to $15 \%$ attributable risk of child behavioural problems related to antenatal anxiety and stress. ${ }^{14}$

\section{Comorbid maternal depression and anxiety}

The importance of comorbid maternal depression and anxiety has been highlighted in several studies. An Australian study found that a third of pregnant and postnatal women with major depression had comorbid anxiety. ${ }^{73}$ In a US population-based study incorporating 4451 postpartum women, a third of women with anxiety symptoms also reported depressive symptoms. ${ }^{74}$ Assessing comorbidity is important because research with non-postnatal populations has shown that comorbid depression and anxiety manifests into more severe symptoms with poorer acute and long-term outcomes, ${ }^{75}$ is more difficult to treat than each disorder alone, ${ }^{76}$ increases the risk for suicide ${ }^{77}$ and requires specific treatment strategies for both sets of symptoms. ${ }^{75}$ The US Task Force for Prevention Screening now endorses screening for perinatal depression, ${ }^{78}$ however, not identifying anxiety symptoms as well underestimates the prevalence of mental health disorders and the need for perinatal mental health services. Matthey et $a l^{79}$ suggests there is a 'hierarchical diagnostic custom' where depression takes precedence in clinical practice even when anxiety symptoms are a prominent feature. This focus on depression can result in individuals with anxiety (but without depression) being undetected and untreated.

\section{Trait anxiety}

Finally, trait anxiety, a condition clinically different from state anxiety symptoms, refers to the tendency to report negative emotions such as fears and worries across situations and is characterised by a stable perception of environmental stimuli as threatening. In this review, trait anxiety was high with prevalence rates ranging from 29 to $33 \%$ antenatally and decreasing to $23 \%$ postnatally. Although rarely examined, antenatal trait anxiety has been associated with increased risk for preterm birth among African American women. ${ }^{80}$ If trait anxiety is an enduring maternal characteristic then its impact on the child is also likely to continue postnatally. This notion is supported in several studies. In a prospective US study with pregnant women, increasing trait anxiety was associated with poorer overall infant cognition. ${ }^{81}$ In an Australian study, trait anxiety was a predictor of maternal report of difficult infant temperament at 4-6 months postpartum. ${ }^{82}$ Further, a German study found trait anxiety was significantly correlated with impaired maternal bonding. ${ }^{49}$ These results suggest that maternal trait anxiety may be as important as state anxiety symptoms or disorders and warrants further investigation. Antenatal psychological treatment interventions such as cognitive behavioural therapy may optimise child outcomes. ${ }^{82}$ Further, treating maternal trait anxiety may be an important step to determine whether reducing trait anxiety has a direct effect on preterm birth risk. ${ }^{80}$

\section{Implications}

The prevalence of maternal anxiety in the antenatal and postnatal periods were estimated among 221974 women from 34 countries. Results suggest anxiety across the perinatal period is highly prevalent and merits clinical attention similar to that given to perinatal depression. Prevalence rates were significantly higher in low- to middle-income countries possibly indicating cultural influences. The Developmental Origins of Health and Disease paradigm $(\mathrm{DOHaD})^{83}$ suggests that human health and development have their origin in early life from conception to early childhood. During this period, the interplay between maternal and environmental factors programme fetal and child development through physiological changes that have long-lasting consequences on later health. Research to develop evidence-based interventions to reduce fetal and child exposure to risk factors such as perinatal anxiety is warranted in order to promote healthy child development.

\section{Cindy-Lee Dennis, PhD, Lawrence S. Bloomberg Faculty of Nursing, University of Toronto, Toronto, and Li Ka Shing Knowledge Institute, St Michael's Hospital, Toronto, Canada; Kobra Falah-Hassani, PhD, Lawrence S. Bloomberg Faculty of Nursing, University of Toronto, Toronto, Canada; Rahman Shiri, MD, PhD, Finnish Institute of Occupational Health, Helsinki, Finland \\ Correspondence: Cindy-Lee Dennis, University of Toronto, 155 College Street Suite 130, Toronto, ON M5T 1P8, Canada. Email: cindylee.dennis@utoronto.ca \\ First received 3 May 2016, final revision 3 Sep 2016, accepted 13 Nov 2016}

\section{Funding}

We thank Lawrence S. Bloomberg Faculty of Nursing of University of Toronto for providing the Tom Kierans International Postdoctoral Fellowship to K.F.-H.

\section{Acknowledgements}

We thank the following authors for providing additional data: Abiodun O. Adewuya ${ }^{84}$ Mostafa Amr ${ }^{85}$ Marte Helene Bjørk, ${ }^{47}$ Alexa Bonacquisti ${ }^{46}$ Birit F. P. Broekman, ${ }^{13}$ Shayna Cunningham, ${ }^{36}$ Deborah Da Costa, ${ }^{56}$ Janet DiPietro, ${ }^{59}$ Natasa Jokic-Begic, ${ }^{54}$ Susan Garthus-Niegel, ${ }^{3}$ Fragiskos Gonidakis, ${ }^{86}$ Wendy Hall, ${ }^{5}$ Courtney Pierce Keeton, ${ }^{60}$ Sarah Keim, ${ }^{81}$ Sheila W. McDonald, ${ }^{87}$ Barbara Menting, ${ }^{29}$ Khitam Mohammad, ${ }^{58}$ Chiara Pazzagli, ${ }^{57}$ Chantal Razurel, ${ }^{88}$ Patricia H. C. Rondó, ${ }^{35}$ Annerine Roos, ${ }^{40}$ Anne-Laure Sutter-Dallay, ${ }^{89}$ Heidi Stöckl, ${ }^{90}$ Jan Taylor, ${ }^{91}$ Ana Amelia F. Vilela ${ }^{37}$ and Vincenzo Zanardo. ${ }^{92}$

\section{References}

1 Stein A, Pearson RM, Goodman SH, Rapa E, Rahman A, McCallum M, et al. Effects of perinatal mental disorders on the fetus and child. Lancet 2014; 384: $1800-19$.

2 Howard LM, Molyneaux E, Dennis CL, Rochat T, Stein A, Milgrom J. Non-psychotic mental disorders in the perinatal period. Lancet 2014; 384: 1775-88.

3 Garthus-Niegel S, von Soest T, Knoph C, Simonsen TB, Torgersen L, Eberhard-Gran M. The influence of women's preferences and actual mode of delivery on post-traumatic stress symptoms following childbirth: a population-based, longitudinal study. BMC Pregnancy Childbirth 2014; 14: 191.

4 Falah-Hassani K, Shiri R, Dennis CL. Prevalence and risk factors for comorbid postpartum depressive symptomatology and anxiety. J Affect Disord 2016; 198: $142-7$.

5 Hall WA, Hauck YL, Carty EM, Hutton EK, Fenwick J, Stoll K. Childbirth fear, anxiety, fatigue, and sleep deprivation in pregnant women. J Obstet Gynecol Neonatal Nurs 2009; 38: 567-76.

6 Rubertsson C, Hellstrom J, Cross M, Sydsjo G. Anxiety in early pregnancy: prevalence and contributing factors. Arch Womens Ment Health 2014; 17: 221-8.

7 George A, Luz RF, De Tychey C, Thilly N, Spitz E. Anxiety symptoms and coping strategies in the perinatal period. BMC Pregnancy Childbirth 2013; 13: 233.

8 Micali N, Simonoff E, Treasure J. Pregnancy and post-partum depression and anxiety in a longitudinal general population cohort: the effect of eating disorders and past depression. J Affect Disord 2011; 131: 150-7.

9 Farias DR, Pinto TJP, Teofilo MMA, Vilela AAF, Vaz JS, Nardi AE, et al. Prevalence of psychiatric disorders in the first trimester of pregnancy and factors associated with current suicide risk. Psychiatry Res 2013; 210: 962-8.

10 Ibanez G, Charles MA, Forhan A, Magnin G, Thiebaugeorges O, Kaminski M, et al. Depression and anxiety in women during pregnancy and neonatal outcome: data from the EDEN mother-child cohort. Early Hum Dev 2012; 88: 643-9.

11 Sanchez SE, Puente GC, Atencio G, Qiu C, Yanez D, Gelaye B, et al. Risk of spontaneous preterm birth in relation to maternal depressive, anxiety, and stress symptoms. J Reprod Med 2013; 58: 25-33. 
12 Berle JO, Mykletun A, Daltveit AK, Rasmussen S, Holsten F, Dahl AA. Neonatal outcomes in offspring of women with anxiety and depression during pregnancy. A linkage study from The Nord-Trondelag Health Study (HUNT) and Medical Birth Registry of Norway. Arch Womens Ment Health 2005; 8: 181-9.

13 Broekman BF, Chan YH, Chong YS, Kwek K, Cohen SS, Haley CL, et al. The influence of anxiety and depressive symptoms during pregnancy on birth size. Paediatr Perinat Epidemiol 2014; 28: 116-26.

14 Glover V. Maternal depression, anxiety and stress during pregnancy and child outcome; what needs to be done. Best Pract Res Clin Obstet Gynaecol 2014; 28: $25-35$.

15 Van den Bergh BR, Mennes M, Oosterlaan J, Stevens V, Stiers P, Marcoen A, et al. High antenatal maternal anxiety is related to impulsivity during performance on cognitive tasks in 14- and 15-year-olds. Neurosci Biobehav Rev 2005; 29: 259-69.

16 Van den Bergh BR, Marcoen A. High antenatal maternal anxiety is related to ADHD symptoms, externalizing problems, and anxiety in 8- and 9-year-olds. Child Dev 2004; 75: 1085-97.

17 O'Connor TG, Heron J, Glover V, ALSPAC Study Team. Antenatal anxiety predicts child behavioral/emotional problems independently of postnatal depression. J Am Acad Child Adolesc Psychiatry 2002; 41: 1470-7.

18 Van Batenburg-Eddes T, Brion MJ, Henrichs J, Jaddoe VW, Hofman A, Verhulst FC, et al. Parental depressive and anxiety symptoms during pregnancy and attention problems in children: a cross-cohort consistency study. J Child Psychol Psychiatry 2013; 54: 591-600.

19 McLeod BD, Wood JJ, Weisz JR. Examining the association between parenting and childhood anxiety: a meta-analysis. Clin Psychol Rev 2007; 27: 155-72.

20 Barker ED, Jaffee SR, Uher R, Maughan B. The contribution of prenatal and postnatal maternal anxiety and depression to child maladjustment. Depress Anxiety 2011; 28: 696-702.

21 Bögels SM, Brechman-Toussaint ML. Family issues in child anxiety: attachment, family functioning, parental rearing and beliefs. Clin Psychol Rev 2006; 26: 834-56.

22 Williams SR, Kertz SJ, Schrock MD, Woodruff-Borden J. A sequential analysis of parent-child interactions in anxious and nonanxious families. J Clin Child Adolesc Psychol 2012; 41: 64-74.

23 Joussemet M, Vitaro F, Barker ED, Cote S, Nagin DS, Zoccolillo M, et al. Controlling parenting and physical aggression during elementary school. Child Dev 2008; 79: 411-25.

24 Moher D, Liberati A, Tetzlaff J, Altman DG, Group P. Preferred reporting items for systematic reviews and meta-analyses: the PRISMA statement. PLOS Med 2009; 6: e1000097.

25 Armijo-Olivo S, Stiles CR, Hagen NA, Biondo PD, Cummings GG. Assessment of study quality for systematic reviews: a comparison of the Cochrane Collaboration Risk of Bias Tool and the Effective Public Health Practice Project Quality Assessment Tool: methodological research. J Eval Clin Pract 2012; 18: 12-8.

26 Higgins J, Green S. Cochrane Handbook for Systematic Reviews of Interventions Version 5.1.0 (updated March 2011). The Cochrane Collaboration, 2009 (http://handbook.cochrane.org/).

27 Higgins JP, Thompson SG. Quantifying heterogeneity in a meta-analysis. Stat Med 2002; 21: 1539-58.

28 Betts KS, Williams GM, Najman JM, Alati R. Maternal depressive, anxious, and stress symptoms during pregnancy predict internalizing problems in adolescence. Depress Anxiety 2014; 31: 9-18.

29 Huizink AC, Menting B, Oosterman M, Verhage ML, Kunseler FC, Schuengel C. The interrelationship between pregnancy-specific anxiety and general anxiety across pregnancy: a longitudinal study. J Psychosom Obstet Gynaecol 2014; 35: 92-100.

30 Meijer JL, Bockting CL, Stolk RP, Kotov R, Ormel J, Burger H. Associations of life events during pregnancy with longitudinal change in symptoms of antenatal anxiety and depression. Midwifery 2014; 30: 526-31.

31 Makara-Studzinska M, Morylowska-Topolska J, Sygit K, Sygit M, Gozdziewska M. Socio-demographical and psychosocial determinants of anxiety symptoms in a population of pregnant women in the regions of central and eastern Poland. Ann Agric Environ Med 2013; 20: 195-202.

32 Figueiredo $B$, Conde $A$. Anxiety and depression in women and men from early pregnancy to 3-months postpartum. Arch Womens Ment Health 2011; 14: 247-55.

33 Goebert D, Morland L, Frattarelli L, Onoye J, Matsu C. Mental health during pregnancy: a study comparing Asian, Caucasian and Native Hawaiian women. Matern Child Health J 2007; 11: 249-55.

34 Lee AM, Lam SK, Sze Mun Lau SM, Chong CS, Chui HW, Fong DY. Prevalence, course, and risk factors for antenatal anxiety and depression. Obstet Gynecol 2007; 110: 1102-12.
35 Rondo PH, Ferreira RF, Nogueira F, Ribeiro MC, Lobert H, Artes R. Maternal psychological stress and distress as predictors of low birth weight, prematurity and intrauterine growth retardation. Eur J Clin Nutr 2003; 57: 266-72.

36 Rosenthal L, Earnshaw VA, Lewis TT, Reid AE, Lewis JB, Stasko EC, et al. Changes in experiences with discrimination across pregnancy and postpartum: age differences and consequences for mental health. Am J Public Health 2015; 105: 686-93.

37 Vilela AA, Pinto TJ, Rebelo F, Benaim C, Lepsch J, Dias-Silva $\mathrm{CH}$, et al. Association of prepregnancy dietary patterns and anxiety symptoms from midpregnancy to early postpartum in a prospective cohort of Brazilian women. J Acad Nutr Diet 2015; 115: 1626-35.

38 Khashan AS, Everard C, McCowan LM, Dekker G, Moss-Morris R, Baker PN et al. Second-trimester maternal distress increases the risk of small for gestational age. Psychol Med 2014; 44: 2799-810.

39 Fadzil A, Balakrishnan K, Razali R, Sidi H, Malapan T, Japaraj RP, et al. Risk factors for depression and anxiety among pregnant women in Hospital Tuanku Bainun, Ipoh, Malaysia. Asia Pac Psychiatry 2013; 5 (suppl 1): 7-13.

40 Roos A, Faure S, Lochner C, Vythilingum B, Stein DJ. Predictors of distress and anxiety during pregnancy. Afr J Psychiatry 2013; 16: 118-122.

41 Couto ER, Couto E, Vian B, Gregório Z, Nomura ML, Zaccaria R, et al. Quality of life, depression and anxiety among pregnant women with previous adverse pregnancy outcomes. Sao Paulo Med J 2009; 127: 185-9.

42 van Batenburg-Eddes T, de Groot L, Huizink AC, Steegers EA, Hofman A, Jaddoe VW, et al. Maternal symptoms of anxiety during pregnancy affect infant neuromotor development: the generation R study. Dev Neuropsychol 2009; 34: 476-93.

43 Shi SX, Tang YF, Cheng LN, Su QF, Qi K, Yang YZ. An investigation of the prevalence of anxiety or depression and related risk factors in women during pregnancy and postpartum. Chin Ment Health J 2007; 21: 254-8.

44 Heron J, O'Connor TG, Evans J, Golding J, Glover V, Team AS. The course of anxiety and depression through pregnancy and the postpartum in a community sample. J Affect Disord 2004; 80: 65-73.

45 Dole N, Savitz DA, Hertz-Picciotto I, Siega-Riz AM, McMahon MJ, Buekens P. Maternal stress and preterm birth. Am J Epidemiol 2003; 157: 14-24.

46 Aaron E, Bonacquisti A, Geller PA, Polansky M. Perinatal depression and anxiety in women with and without human immunodeficiency virus infection. Womens Health Issues 2015; 25: 579-85.

47 Bjork MH, Veiby G, Reiter SC, Berle JO, Daltveit AK, Spigset O, et al. Depression and anxiety in women with epilepsy during pregnancy and after delivery: a prospective population-based cohort study on frequency, risk factors, medication, and prognosis. Epilepsia 2015; 56: 28-39.

48 Cheng TS, Chen $\mathrm{H}$, Lee T, Teoh $\mathrm{OH}$, Shek LP, Lee BW, et al. An independent association of prenatal depression with wheezing and anxiety with rhinitis in infancy. Pediatr Allergy Immunol 2015; 26: 765-71.

49 Dubber S, Reck C, Muller M, Gawlik S. Postpartum bonding: the role of perinatal depression, anxiety and maternal-fetal bonding during pregnancy. Arch Womens Ment Health 2015; 18: 187-95.

50 Ibanez G, Bernard JY, Rondet C, Peyre H, Forhan A, Kaminski M, et al. Effects of antenatal maternal depression and anxiety on children's early cognitive development: a prospective cohort study. PLOS One 2015; 10: e0135849.

51 Pazzagli C, Laghezza L, Capurso M, Sommella C, Lelli F, Mazzeschi C. Antecedents and consequences of fear of childbirth in nulliparous and parous women. Infant Ment Health J 2015; 36: 62-74.

52 Pisoni C, Garofoli F, Tzialla C, Orcesi S, Spinillo A, Politi P, et al. Complexity of parental prenatal attachment during pregnancy at risk for preterm delivery. J Matern Fetal Neonatal Med 2016; 29: 771-6.

53 Ferreira CR, Orsini MC, Vieira CR, do Amarante Paffaro AM, Silva RR. Prevalence of anxiety symptoms and depression in the third gestational trimester. Arch Gynecol Obstet 2015; 291: 999-1003.

54 Jokic-Begic N, Zigic L, Nakic Rados S. Anxiety and anxiety sensitivity as predictors of fear of childbirth: different patterns for nulliparous and parous women. J Psychosom Obstet Gynaecol 2014; 35: 22-8.

55 Tan PC, Zaidi SN, Azmi N, Omar SZ, Khong SY. Depression, anxiety, stress and hyperemesis gravidarum: temporal and case controlled correlates. PLOS One 2014; 9: e92036.

56 Verreault N, Da Costa D, Marchand A, Ireland K, Dritsa M, Khalife S. Rates and risk factors associated with depressive symptoms during pregnancy and with postpartum onset. J Psychosom Obstet Gynaecol 2014; 35: 84-91.

57 Bindt C, Guo N, Bonle MT, Appiah-Poku J, Hinz R, Barthel D, et al. No association between antenatal common mental disorders in low-obstetric risk women and adverse birth outcomes in their offspring: results from the CDS study in Ghana and Cote D'Ivoire. PLOS One 2013; 8: e80711. 
58 Mohammad KI, Gamble J, Creedy DK. Prevalence and factors associated with the development of antenatal and postnatal depression among Jordanian women. Midwifery 2011; 27: e238-45.

59 Dipietro JA, Costigan KA, Sipsma HL. Continuity in self-report measures of maternal anxiety, stress, and depressive symptoms from pregnancy through two years postpartum. J Psychosom Obstet Gynaecol 2008; 29: 115-24.

60 Keeton CP, Perry-Jenkins M, Sayer AG. Sense of control predicts depressive and anxious symptoms across the transition to parenthood. J Fam Psychol 2008; 22: 212-21.

61 Ross LE, Gilbert Evans SE, Sellers EM, Romach MK. Measurement issues in postpartum depression part 1: anxiety as a feature of postpartum depression. Arch Womens Ment Health 2003; 6: 51-7.

62 Teixeira JM, Fisk NM, Glover V. Association between maternal anxiety in pregnancy and increased uterine artery resistance index: cohort based study. BMJ 1999; 318: 153-7

63 Crandon AJ. Maternal anxiety and obstetric complications. J Psychosom Res 1979; 23: 109-11.

64 Alonso J, Lepine JP, Committee ESMS. Overview of key data from the European Study of the Epidemiology of Mental Disorders (ESEMeD). J Clin Psychiatry 2007; 68 (suppl 2): 3-9.

65 Wittchen HU, Jacobi F. Size and burden of mental disorders in Europe - a critical review and appraisal of 27 studies. Eur Neuropsychopharmacol 2005 15: 357-76.

66 Meades R, Ayers S. Anxiety measures validated in perinatal populations: a systematic review. J Affect Disord 2011; 133: 1-15.

67 Gavin NI, Gaynes BN, Lohr KN, Meltzer-Brody S, Gartlehner G, Swinson T. Perinatal depression: a systematic review of prevalence and incidence. Obstet Gynecol 2005; 106: 1071-83.

68 Bandelow B, Michaelis S. Epidemiology of anxiety disorders in the 21st century. Dialogues Clin Neurosci 2015; 17: 327-35.

69 Fisher J, Cabral de Mello M, Patel V, Rahman A, Tran T, Holton S, et al. Prevalence and determinants of common perinatal mental disorders in women in low- and lower-middle-income countries: a systematic review. Bull World Health Organ 2012; 90: 139G-49G.

70 Kessler RC, Demler O, Frank RG, Olfson M, Pincus HA, Walters EE, et al. Prevalence and treatment of mental disorders, 1990 to 2003. N Engl J Med 2005; 352: 2515-23.

71 Wittchen HU, Jacobi F, Rehm J, Gustavsson A, Svensson M, Jonsson B, et al The size and burden of mental disorders and other disorders of the brain in Europe 2010. Eur Neuropsychopharmacol 2011; 21: 655-79.

72 Alonso J, Angermeyer MC, Bernert S, Bruffaerts R, Brugha TS, Bryson $\mathrm{H}$, et al. 12-Month comorbidity patterns and associated factors in Europe: results from the European Study of the Epidemiology of Mental Disorders (ESEMeD) project. Acta Psychiatr Scand Suppl 2004; 109: 28-37.

73 Austin MP, Hadzi-Pavlovic D, Priest SR, Reilly N, Wilhelm K, Saint K, et al. Depressive and anxiety disorders in the postpartum period: how prevalent are they and can we improve their detection? Arch Womens Ment Health 2010; 13: 395-401.

74 Farr SL, Dietz PM, O'Hara MW, Burley K, Ko JY. Postpartum anxiety and comorbid depression in a population-based sample of women. $J$ Womens Health (LarChmt) 2014; 23: 120-8.

75 Rivas-Vazquez RA, Saffa-Biller D, Ruiz I, Blais MA, Rivas-Vazquez A. Current issues in anxiety and depression: Comorbid, mixed and subthreshold disorders. Prof Psychol Res Pract 2004; 35: 74-83.
76 Emmanuel J, Simmonds S, Tyrer P. Systematic review of the outcome of anxiety and depressive disorders. Br J Psychiatry 1998; 173 (suppl 34): $35-41$.

77 Fawcett J. The detection and consequences of anxiety in clinical depression. J Clin Psychiatry 1997; 58 (suppl 8): 35-40.

78 Siu AL, US Preventative Services Task Force, Bibbins-Domingo K, Grossman DC, Baumann LC, Davidson KW, et al. Screening for depression in adults: US Preventive Services Task Force Recommendation Statement. JAMA 2016; 315: 380-7.

79 Matthey S, Barnett B, Howie P, Kavanagh DJ. Diagnosing postpartum depression in mothers and fathers: whatever happened to anxiety? J Affect Disord 2003; 74: 139-47.

80 Catov JM, Abatemarco DJ, Markovic N, Roberts JM. Anxiety and optimism associated with gestational age at birth and fetal growth. Matern Child Health J 2010; 14: 758-64.

81 Keim SA, Daniels JL, Dole N, Herring AH, Siega-Riz AM, Scheidt PC. A prospective study of maternal anxiety, perceived stress, and depressive symptoms in relation to infant cognitive development. Early Hum Dev 2011; 87: $373-80$

82 Austin MP, Hadzi-Pavlovic D, Leader L, Saint K, Parker G. Maternal trait anxiety, depression and life event stress in pregnancy: relationships with infant temperament. Early Hum Dev 2005; 81: 183-90.

83 Heindel JJ, Vandenberg LN. Developmental origins of health and disease: a paradigm for understanding disease cause and prevention. Curr Opin Pediatr 2015; 27: 248-53.

84 Adewuya AO, Afolabi OT. The course of anxiety and depressive symptoms in Nigerian postpartum women. Arch Womens Ment Health 2005; 8: 257-9.

85 Amr MA, Hussein Balaha MH. Minor psychiatric morbidity in young Saudi mothers using Mini International Neuropsychiatric Interview (MINI). J Coll Physicians Surg Pak 2010; 20: 680-4.

86 Gonidakis F, Rabavilas AD, Varsou E, Kreatsas G, Christodoulou GN. Maternity blues in Athens, Greece: a study during the first 3 days after delivery. J Affect Disord 2007; 99: 107-15.

87 McDonald SW, Kingston D, Bayrampour H, Dolan SM, Tough SC. Cumulative psychosocial stress, coping resources, and preterm birth. Arch Womens Ment Health 2014; 17: 559-68.

88 Razurel C, Kaiser B. The role of satisfaction with social support on the psychological health of primiparous mothers in the perinatal period. Women Health 2015; 55: 167-86.

89 Sutter-Dallay AL, Giaconne-Marcesche V, Glatigny-Dallay E, Verdoux H. Women with anxiety disorders during pregnancy are at increased risk of intense postnatal depressive symptoms: a prospective survey of the MATQUID cohort. Eur Psychiatry 2004; 19: 459-63.

90 Mahenge B, Stockl H, Likindikoki S, Kaaya S, Mbwambo J. The prevalence of mental health morbidity and its associated factors among women attending a prenatal clinic in Tanzania. Int J Gynaecol Obstet 2015; 130: 261-5.

91 Taylor J, Johnson M. The role of anxiety and other factors in predicting postnatal fatigue: from birth to 6 months. Midwifery 2013; 29: 526-34.

92 Zanardo V, Gasparetto S, Giustardi A, Suppiej A, Trevisanuto D, Pascoli I, et al. Impact of anxiety in the puerperium on breast-feeding outcomes: role of parity. J Pediatr Gastroenterol Nutr 2009; 49: 631-4. 